\title{
A Survey of BL Herculis-Type Models
}

\author{
J. Robert Buchler and Pawel Moskalik \\ Department of Physics, University of Florida, \\ Gainesville, Florida 32611, U.S.A.
}

\begin{abstract}
We have studied the nonlinear behavior of several sequences of BL Herculistype models. The question arose whether the $2: 1$ resonance between the fundamental mode and the second overtone would cause the same systematic variation of Fourier parameters of the pulsation cycle with period ratio $P_{2} / P_{0}$ as was seen in the classical Cepheids. We find that for the BL Her stars, the behaviour of the light-curve Fourier phases is markedly different from the Cepheid case. In particular, $\phi_{21}$ exhibits essentially a featureless, monotone increase throughout the range of $P_{2} / P_{0}$, which is in qualitative agreement with the observed trend (Petersen \& Diethelm 1986). In the velocity curves, on the other hand, the 2:1 resonance is a dominant feature and the progression of the Fourier phases and the amplitude ratios is similar to those witnessed in the Cepheids. However, here the sensitivity to the stellar masses and luminosities is significantly stronger. Our results show that radial velocity observations of the BL Her stars would pinpoint the resonance and put important new constraints on the models.
\end{abstract}

